\title{
Sequential Cooperative Spectrum Sensing Technique in Time Varying Channel
}

\author{
Warit Prawatmuang, Daniel K. C. So, Member, IEEE and Emad Alsusa, Senior Member, IEEE
}

\begin{abstract}
Cognitive radio opportunistically accesses the spectrum while the licensed user is idle. A spectrum sensing procedure to monitor primary users' existence is therefore vital to cognitive radios. In this paper, we investigate the energy detection based sequential cooperative spectrum sensing technique in time varying channels. By utilizing past local observations from previous sensing slots, cognitive radio nodes can aggregate the current and previously received energy values to improve the detection performance. We propose the weighted sequential energy detector (SED) in which a fixed number of past observations are taken for decision making. In addition, we propose two adaptive schemes, namely the Two-Stage SED and the Differential SED, where a cognitive radio user uses previously received energy values until it detects a change in primary user's activity. The probability of false alarm and detection for the Weighted SED and Two-Stage SED schemes are derived. The results show that the proposed schemes achieve better detection performance over the conventional cooperative energy detection technique. In particular, the Two-Stage SED and Differential SED schemes can satisfy the IEEE 802.22 standard's requirement of $10 \%$ false alarm and $90 \%$ detection in scenarios that conventional techniques cannot accommodate.
\end{abstract}

Index Terms-Cognitive Radio, Spectrum Sensing, Sequential Spectrum Sensing, Adaptive Spectrum Sensing

\section{INTRODUCTION}

Wireless networks have been regulated by the fixed spectrum assignment policy, in which their usage are limited to a certain part of the spectrum. However, current studies have found that the rest of the spectrum remains under-utilized [1]. With the high demand of mobile services, new approaches that efficiently utilize the spectrum are needed. The Federal Communication Commission in the US approved the use of TV white space by unlicensed wireless devices through a geolocation database [2]. While this approach improves spectrum usage in the TV white space, it will not fully utilize other part of the spectrum. It will also not meet the demand of future networks, which will have high density base station deployment and efficient self organizing capability [3]. By opportunistically accessing the licensed spectrum without interfering with the licensed users, Cognitive Radio (CR)

Manuscript received August 1, 2013; revised November 23, 2013 and February 16, 2014. The editor coordinating the review of this paper and approving it for publication was Dr. Manav Bhatnagar.

This paper was presented in part at the IEEE Vehicular Technology Conference (VTC2012-Spring), Yokohama, Japan, May 2012 and at the IEEE International Symposium on Personal, Indoor and Mobile Radio Communications (PIMRC'12), Sydney, Australia, September 2012.

The authors are with the School of Electrical and Electronic Engineering, The University of Manchester, United Kingdom. Email: warit.prawatmuang @gmail.com,d.so@manchester.ac.uk, e.alsusa@manchester.ac.uk.

Digital Object Identifier improves the efficiency of spectrum usage by first sensing for the existence of Primary User (PU) transmissions [4]. If the spectrum is vacant, the CR transmitter will start communicating to its receiver. When the PUs retransmit again, the CR users stop their transmission immediately to avoid creating interference to the PUs. Because of its flexibility, CR is considered as a key enabler for the $5^{\text {th }}$ Generation $(5 \mathrm{G})$ mobile network [5].

Spectrum sensing is vital for CR. Various spectrum sensing techniques in CR literature are reviewed in [6]-[9]. Matchedfiltering is the optimal method when information about PU's transmitted signal is known to the CR user. However, when prior knowledge on the PU's signal is not available, energy detection is favourable due to its low computational and implementation complexities.

Spectrum sensing is based on the CR user's observation. When the CR user experiences a deep fade or shadowing from the licensed transmitter, the hidden terminal problem will occur which leads to missed detection, and causes interference to the PU. In order to tackle this problem, cooperative detection has been proposed to gather sensing information from multiple CR users [10]. Each CR user senses the spectrum and forwards its local observation to the fusion centre. Based on multiple observations, the fusion centre will then decide whether the licensed user exists or not. As users are spatially separated, cooperative detection can exploit spatial diversity and minimizes the uncertainty in single user detection.

To improve the sensing performance, many novel techniques have been investigated and proposed. For instance, in Double Threshold energy detection scheme, CR user forwards its local decision to the fusion center only when it is certain about its decision, otherwise it forwards the local observation to the fusion center [11]-[13]. In terms of detection threshold optimization, constant false-alarm rate detector is to maximize the probability of detection while probability of false alarm is fixed [9]. Differently, under minimum-error-probability criterion, the optimal threshold is obtained as the threshold that minimizes the total probability of error [14], [15]. In addition, the minimum-error-probability criterion with energy detector based cooperative spectrum sensing under imperfect reporting channel was discussed in [16], [17]. Furthermore, sequential spectrum sensing has been introduced in [18] to make use of past observations. A local decision is made when the CR user is confident on its current observation-based on the loglikelihood ratio (LLR). Otherwise, it will not make any local decision but simply adds the new LLR value onto the previous one. Assuming the channel is static, this procedure will improve the LLR and thus improve the detection performance. 
However, computing the LLR involves more computational complexity than the simple energy detector. Moreover, similar to most existing research work on spectrum sensing, they assume static PU activity.

In this paper, we consider intermittent PU activity in the system model to imitate practical scenario. We also propose three sequential cooperative spectrum sensing schemes under this scenario in time varying channel. First, the Weighted Sequential Energy Detector (SED), which applies different weighting onto a fixed number of past observations is proposed to improve the detection performance. Both equal weighting and exponential weighting are investigated. Then, two adaptive schemes, namely the Two-Stage SED and the Differential SED, which adapt themselves to their decision on the PU's state are proposed.

The rest of this paper is organised as follows. In Section II, we present the system model for the sequential sensing procedure and the PU activity model. Then, the three proposed spectrum sensing schemes are presented in Section III. A detailed analysis as well as the generic form for probability of false alarm and detection for Weighted SED and Two-Stage SED scheme is given in Section IV. Simulation results for these schemes are shown and compared with the conventional scheme in Section V. The conclusions of this paper are provided in Section VI.

\section{SySTEM MODEL}

A cooperative spectrum sensing system consists of multiple CR users and a fusion centre. They are operated underneath a separate PU system, which does not cooperate with the CR system for spectrum sharing. The CR users sense the spectrum, and forward their local decisions to the fusion centre. The fusion centre makes the final decision using a certain combining rule, such as OR-rule, AND-rule, and majority vote [19]. This will improve the sensing performance by overcoming fading and hidden terminal problem. For each CR user, the energy detection technique is used [20]. It is a simple and effective approach whereby the received signal energy is compared to a detection threshold to determine the existence of the PUs.

The received energy value at the $\mathrm{CR}$ user can be expressed as

$$
y=\left\{\begin{array}{cc}
\sum_{i=1}^{m}\left|n_{i}\right|^{2} & H_{0} \\
\sum_{i=1}^{m}\left|h_{i} s_{i}+n_{i}\right|^{2} & H_{1}
\end{array}\right.
$$

where $m, h_{i}, s_{i}$ and $n_{i}$ is the time-bandwidth product, channel gain, PU's signal and noise in the $i^{\text {th }}$ time slot and $H_{0}, H_{1}$ represents the case when PU is idle and active respectively. When PU is idle $\left(H_{0}\right)$, the received signal energy at the CR user follows the Central Chi-Squared distribution with $2 m$ degree of freedom. Its probability density function (PDF) can be written as [21]

$$
f\left(y \mid H_{0}\right)=\frac{y^{m-1} e^{-y / 2}}{\Gamma(m) \cdot 2^{m}}
$$

where $\Gamma($.$) is the gamma function. And, the probability of$ false alarm can be given as

$$
\begin{aligned}
P_{f}(\lambda) & =\int_{\lambda}^{\infty} f\left(y \mid H_{0}\right) d y \\
& =\frac{\Gamma(m, \lambda / 2)}{\Gamma(m)}
\end{aligned}
$$

where $\lambda$ is the energy detection threshold.

On the contrary, when a PU is active $\left(H_{1}\right)$, the received signal energy for a particular instantaneous SNR follows the Non-Central Chi-Squared distribution with degree of freedom of $2 m$ and non-centrality parameter of $2 m \gamma$, where $\gamma$ is the instantaneous SNR. Its PDF can be written as [21]

$$
f\left(y, \gamma \mid H_{1}\right)=\frac{y^{m-1} e^{-(y+2 m \gamma) / 2}}{2^{m} \cdot \Gamma(m)}{ }_{0} F_{1}\left(m, \frac{m \gamma y}{2}\right)
$$

where ${ }_{0} F_{1}(.,$.$) is the confluent hypergeometric limit function$ [22].

In fading channels, however, the instantaneous SNR at the $\mathrm{CR}$ user is random. Assuming Rayleigh fading with a PDF of $f_{h}(\gamma)$, the PDF for the received signal energy can be obtained by

$$
f\left(y \mid H_{1}\right)=\int_{0}^{\infty} f\left(y, \gamma \mid H_{1}\right) \cdot f_{h}(\gamma) d \gamma .
$$

After some derivations, we obtain

$$
\begin{aligned}
f\left(y \mid H_{1}\right)= & \frac{e^{-\frac{1}{2(1+m \bar{\gamma})} y}}{2 \cdot(1+m \bar{\gamma}) \cdot\left(\frac{m \bar{\gamma}}{1+m \bar{\gamma}}\right)^{m-1} \cdot \Gamma(m-1)} \\
& \times\left[\Gamma(m-1)-\Gamma\left(m-1, \frac{m \bar{\gamma} y}{2+2 m \bar{\gamma}}\right)\right]
\end{aligned}
$$

where $\bar{\gamma}$ is the average SNR received at the CR. Hence, the probability of detection $\left(P_{d}\right)$ is

$$
\begin{aligned}
P_{d}(\lambda) & =\int_{\lambda}^{\infty} f\left(y \mid H_{1}\right) d y \\
& =\frac{\Gamma(m-1, \lambda / 2)}{\Gamma(m-1)}+e^{-\frac{\lambda}{2(1+m \bar{\gamma})}} \\
& \times\left(1+\frac{1}{m \bar{\gamma}}\right)^{m-1} \times\left[1-\frac{\Gamma\left(m-1, \frac{\lambda m \bar{\gamma}}{2(1+m \bar{\gamma})}\right)}{\Gamma(m-1)}\right] .
\end{aligned}
$$

In this paper, we consider a more practical scenario whereby the channel and PU activity are both time varying. Therefore, there is a correlation between the previous and current received energy value. This channel correlation is affected by Doppler frequency. When the Doppler frequency is low, channel gain changes slowly and the difference between observations is small. Conversely, high Doppler frequency leads to high channel variation, leading to low channel correlation. On the other hand, the PU activity is modelled according to a twostate Markov chain [23] as shown in Fig. 1. The parameter $\alpha$ and $\beta$ represents the probability of PU changing its state from active $\left(H_{1}\right)$ to idle $\left(H_{0}\right)$ and vice versa, while the notation $1-\alpha$ and $1-\beta$ represents the probability of PU remains in active $\left(H_{1}\right)$ and idle $\left(H_{0}\right)$ state respectively. Hence, a smaller $\alpha$ and $\beta$ represents a lower transitional activity and a higher correlation between two consecutive energy value. 


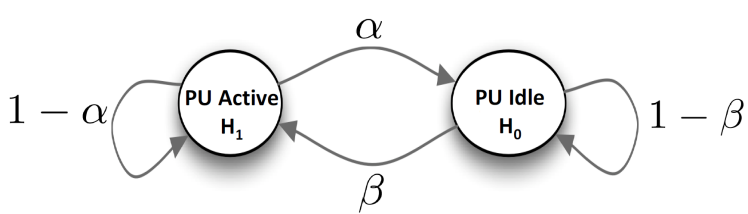

Fig. 1. Two-state Markov chain on primary user's activity model

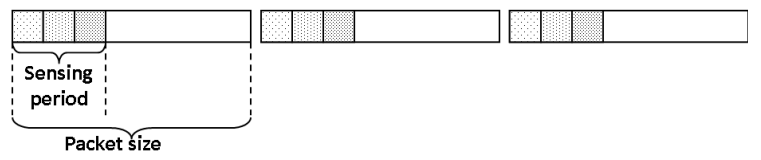

Fig. 2. Sequential sensing procedure

The sequential spectrum sensing technique is used to exploit these correlations. Each CR user senses the spectrum in regular intervals. Instead of deciding the PU's existence only based on the current sensing period, it combines with the previously received energy value to improve detection performance. If the channel is vacant, the CR can use the spectrum before the next sensing period. This procedure is illustrated in Fig. 2. It is clear that if the channel and the PU activity is static, the SED will have very good detection performance.

\section{Proposed Sequential Cooperative Sensing SCHEMES}

The question for using sequential spectrum sensing in time varying channels is how should the previous and current energy values be combined locally. This is especially challenging when the PU has changed its activity as the received signal energy will change significantly. In this section, we discuss our proposed sequential cooperative spectrum sensing schemes. First, we propose the Weighted SED scheme, which simply takes in a fixed number of past observations. Then, two adaptive schemes, namely the Two-Stage SED and Differential SED are proposed. These schemes adaptively determine the number of past observations, based on its own decision on the PU's state.

\section{A. Weighted SED}

The Weighted SED scheme exploits channel correlation by weighting and aggregating current and previous observations. In order not to include outdated observations, we employ the moving average model to combine the energy observations [24]. Then, the weighted output is compared to the threshold locally at the CR node, and the local decision is forwarded to the fusion center. At the fusion centre, we choose the ORrule for global decision making due to its simplicity and PU's protection purpose. Only one-bit CR's decision is forwarded to the fusion centre and if any CR node detects the PU, all CR users will stop the communication to protect the PUs. In other words, this approach makes use of soft combining for local decision and the OR-rule for global decision at the fusion center.
1) Sensing Procedures: First, the energy observations are weighted and aggregated using the moving average model at the $k^{t h}$ sensing slot such that

$$
T_{k}=\mathbf{W} \mathbf{Y}_{k}=\sum_{i=0}^{N-1} w_{i} y_{k-(i+N-1)}
$$

where $T$ is the weighted sum energy, $N$ is number of energy observations taken into the weighting process, and $\mathbf{Y}_{k}$ is the local observation vector at the $k^{t h}$ sensing slot denoted as

$$
\mathbf{Y}_{k}=\left[\begin{array}{llll}
y_{k-(N-1)} & y_{k-(N-2)} & \cdots & y_{k}
\end{array}\right]^{T}
$$

where $y_{k}$ is the current received signal and $y_{k-i}$ is the observation from the $i^{\text {th }}$ sensing slot before. $\mathbf{W}$ is the weighting vector such that

$$
\mathbf{W}=\left[\begin{array}{llll}
w_{0} & w_{1} & \cdots & w_{N-1}
\end{array}\right]
$$

where $w_{i}$ is the $i^{t h}$ weighting factor. $\mathbf{W}$ is a normalized vector such that $\left(w_{0}+w_{1}+\ldots+w_{N-1}\right)=1$.

Then, the decision $d_{k}$ is made by comparing $T_{k}$ to the threshold $\lambda$.

$$
d_{k}=\left\{\begin{array}{l}
H_{0}, \text { if } T_{k}<\lambda \\
H_{1}, \text { otherwise. }
\end{array}\right.
$$

Finally, the local decision $d_{k}$ is sent to the fusion centre to make a final decision on PU's existence using the OR-rule.

2) Weight Computation: The weighting vector $\mathbf{W}$ is a key component of sequential cooperative sensing. Ideally, under minimum-error probability criterion, this vector should be optimized by minimizing the false alarm $\left(P_{f}\right)$ and miss detection probability $\left(P_{m}\right)$ [19]. The optimization criteria then becomes

$$
\mathbf{W}^{*}=\arg \min _{\mathbf{W}}\left(P_{f}+P_{m}\right)
$$

where $P_{f}$ and $P_{d}$ are the complementary cumulative distribution function (CCDF) for weighted sum of $N$-random variables as

$$
P_{f}=P\left(T>\lambda \mid H_{0}\right)
$$

and

$$
P_{d}=P\left(T>\lambda \mid H_{1}\right)
$$

Hence optimizing $\mathbf{W}$ is complicated and the closed-form and optimal solution is difficult to obtain [25].

3) Sub-Optimal Weight Vector: Since the optimal solution is difficult to obtain, a sub-optimal weight calculation approach is proposed here. Considering the PDF of received energy when PU is idle $\left(H_{0}\right)$ and active $\left(H_{1}\right)$ as shown in Fig. 3, the optimal detection approach is to determine the detection threshold that can jointly minimize the false alarm probability $P_{f}$ and missed detection $P_{m}$, i.e., the shaded area in the figure. With SED, we can include past observations to vary the PDF of the weighted sum energy such that the shaded area is minimized. This can be achieved, if possible, by separating the means, or minimizing the variance of the received energy in the $H_{0}$ and $H_{1}$ case. However, we will show first that applying weighting will not be able to vary the mean by the following proposition. 




Fig. 3. Probability density function of received energy when PU is idle $\left(H_{0}\right)$ and active $\left(H_{1}\right)$

Proposition 1: For a normalized weight vector, the mean of the weighted sum energy equals to the mean of the local observation (i.e. $E[T]=E[Y]$ ) if the channel and PU activity is static

Proof:

$$
\begin{aligned}
T_{k} & =\sum_{i=0}^{N-1} w_{i} y_{k-(i+N-1)} \\
E[T] & =w_{0} \cdot E\left[Y_{N-1}\right]+\ldots+w_{N-1} \cdot E\left[Y_{0}\right]
\end{aligned}
$$

where $Y_{0}$ and $Y_{k}$ are the set of current received signal and that of the $k^{t h}$ sensing slot before. Since the channel and PU activity is static, the mean of each local observation is identical. Hence,

$$
E[T]=\left(w_{0}+w_{1}+\ldots+w_{N-1}\right) \cdot E[Y]=E[Y]
$$

The importance of this proposition is that the mean of the weighted sum energy will not be affected by the choice of the weighting vector $\mathbf{W}$, and is the same as the mean of a single observation. In other words, the separation between the mean of received energy in $H_{0}$ and $H_{1}$ case will not be affected by varying $\mathbf{W}$. Hence, to minimize the shaded area, the other option is to reduce the variance of these PDFs. The effect of variance on the distribution of received energy in $H_{0}$ and $H_{1}$ case is illustrated in Fig. 4. When more observations are taken, the variance for both received energy in two cases will be reduced. Hence, the separation between the distribution in $H_{0}$ and $H_{1}$ case is more evident and this leads to better detection performance. We also show that when equal weighting is applied, the variance decreases proportionally to the number of samples taken in the Appendix.

4) Weight Vector for Static PU Activity: As discussed above, the optimal weighting is one that can minimize the variance of $T$ in both $H_{0}$ and $H_{1}$ case. However, its complexity involves minimizing both distributions at the same time. Hence, we propose a sub-optimal approach to obtain the weighting vector by minimizing the variance of weighted

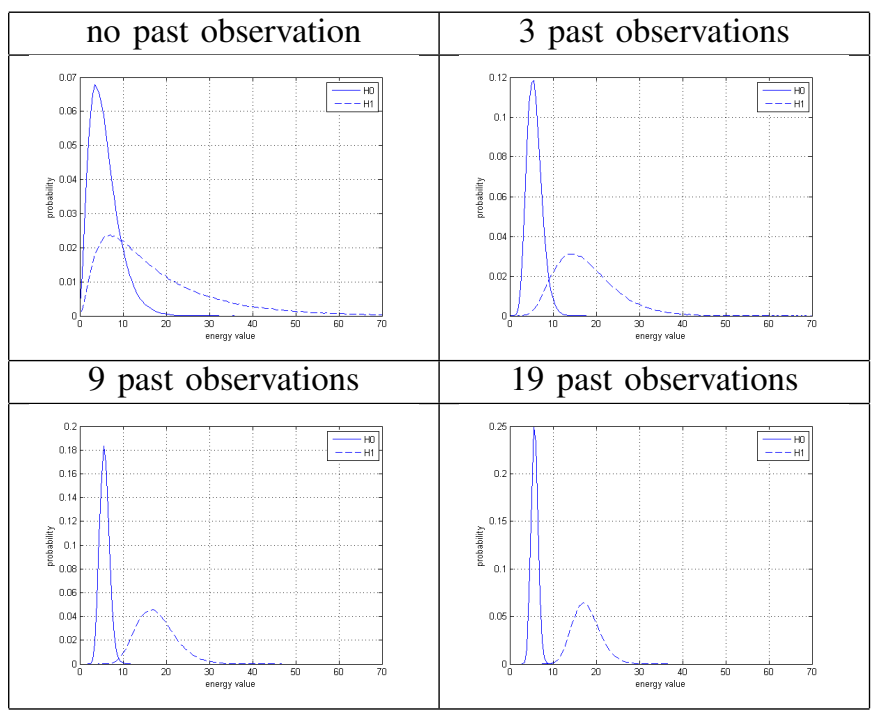

Fig. 4. Energy distribution for weighted local observation with different number of past observations taken

received energy in $H_{0}$ or $H_{1}$ case separately. When the PU activity is static (i.e. the PU is either always on or always off), the following can be defined.

Proposition 2: In quasi-static fading channel, the optimal weight vector that minimizes the variance of received energy in $H_{0}$ and $H_{1}$ is the equal weighting.

Proof: In quasi-static fading case, there is no correlation between each observation and hence the variance, which is derived in the Appendix, becomes

$$
\operatorname{Var}(T)=\sum_{i=0}^{N-1} w_{i}^{2} \cdot \operatorname{Var}\left(Y_{i+N-1}\right) .
$$

Since the PU activity is assumed to be static, the variance of the energy observations are identical. Thus the optimization criteria to minimize the variance of $T$ is

$$
\begin{aligned}
& \operatorname{Min} \sum_{i=1}^{N} w_{i}^{2} \\
& \text { s.t. }\left(w_{1}+w_{2}+\ldots+w_{N}\right)=1 .
\end{aligned}
$$

By using standard Lagrange multiplier, the solution is

$$
w_{A}=w_{B}=\ldots=w_{N}=\frac{1}{N} .
$$

Hence, in quasi-static fading channel, equal weighting is optimal as it gives the lowest variance.

Corollary 1: In time-varying channel, the optimal weight vector that minimizes the variance of $H_{0}$ is the equal weighting.

Proof: As the PU does not exist in $H_{0}$, the channel will not affect the received energy observation. Hence, the proof follows that of Proposition 2.

Now the remaining problem is the optimization of weight vector for $H_{1}$ in time varying channel. This involves the computation of covariance between observations and a goal is to minimize (33), which is the variance of $T$. This minimization is a convex optimization problem and the solution could be obtained by using Lagrange multiplier, provided that 
the covariance matrix is known. However, it will be shown in Section $\mathrm{V}$ that equal weighting outperforms the optimized weight vector based on $H_{1}$.

5) Weight Vector for Intermittent PU Activity: When PU activity is varying, the received energy value at the CR could change suddenly between observations. Hence, a practical approach is to rely more on the newer observations for detection. Thus, we propose the use of a simple exponential weighting, whereby each past observation is weighted less by a factor of $\frac{1}{e}$

$$
\mathbf{W}=\frac{1}{K}\left[\begin{array}{llll}
e^{1} & e^{2} & \ldots & e^{N}
\end{array}\right]
$$

where $K=\sum_{i=1}^{N} e^{i}$ is the normalization factor.

6) Simulation Results: Simulation results, the more details of which will be discussed in Section V, show that the proposed Weighted SED scheme can improve the detection performance by utilizing past observations. It provides significantly better detection performance than the conventional OR-rule energy detection scheme, when PU is static. However, when PU is intermittent, simulation result shows that the Weighted SED with equal weighting vector is worsened due to PU activity, especially when it varies a lot. This is due to the heavy reliance on past observations which are already outdated, i.e. PU has already changed its state.

\section{B. Two-Stage SED}

As briefly discussed above, the Weighted SED detection performance will degrade when using outdated past observations, which arises when PU is intermittent and regularly changes state. In this section, we consider an adaptive technique which does not set a fixed number of previous observations but adaptively determine the number of past observation used for the weight combination. The fundamental concept is that if the PU activity has not changed, past observations should be used for weighting to improve performance. However, if the PU has changed its state, past observations should be discarded as they will lead to erroneous detection. Hence, the adaptive SED approaches will need to know when the PU has changed its activity. As CR users perform blind detection and cannot precisely know PU's state, the CR's local observation will be used to perform an initial estimation. With the transition of PU activity determined, the CR user then decides how many past observations it should rely on and take into the weighting process. The current and previous observations are then combined before compared to the threshold locally at the CR node.

Here, we propose an adaptive Two-Stage SED scheme, which as the name suggests, consists of a two stage energy detection procedure. In the first stage, $\mathrm{CR}$ users perform simple single threshold energy detection to decide PU's existence. Based on this sensing decision, CR users estimate whether PU has changed its activity and decide how many past observations to be taken for equal weighting. If the CR users think that a PU has not changed its state, it will include this energy observation value into the weighting procedure. The second stage is then the previously mentioned Weighted SED to determine the existence of PU.

The detailed sensing procedure for Two Stage SED is as follows.

1) First, each $\mathrm{CR}$ users locally perform a conventional sensing by comparing the instantaneous received energy observations to the first stage threshold $\lambda_{1}$.

2) Based on the sensing result in the first stage, CR users determine the number of past observations to be taken into the weight computation. Only the past local decisions that are the same as this initial detection (i.e., the PU presumably maintained its activity) will be used for the weighting procedure. In other words, once the first stage sensing decision is made and is different to previous decisions, all past observations will be discarded as they think that PU has changed its state.

3) To avoid CR users relying on too many past observations and degrade the performance, we limit the number of past observations to be taken in to weight computation to a certain number. $\mathrm{CR}$ users then apply equal weighting to all energy observations that are taken into weight computation in the second stage. The weighted observations are then aggregated similarly to the Weighted SED scheme as in (8).

4) The local decision $d_{k}$ is made by comparing $T_{k}$ to the second stage threshold $\lambda_{2}$.

$$
d_{k}=\left\{\begin{array}{c}
H_{0}, \text { if } T_{k}<\lambda_{2} \\
H_{1}, \text { otherwise. }
\end{array}\right.
$$

5) Finally, the local decision $d_{k}$ is sent to the fusion centre to make a final decision on PU's existence using the OR-rule.

\section{Differential SED}

The Two-Stage SED performs an instantaneous sensing decision and compares with previous decisions to determine the number of included observations. Here, we propose another approach called the Differential SED. In a slow varying fading channel, the channel gain for consecutive sensing slots are not much different. Hence, the received energy value for those consecutive sensing slots would be similar as well, provided that the PU does not change its state. On the contrary, when PU changes its state, the difference between consecutive observations would be high. In this scheme, CR users estimate the PU's state based on this difference. If the difference between two consecutive energy observations is less than a certain threshold, the CR system will consider that the state of PU remains unchanged. As a result, past observations will be used together with the new observation to perform detection. On the other hand, if the difference between consecutive observations are larger than the threshold, the CR system will consider the PU has changed its state. Hence, all previous observations will be discarded and the detection will be based only on this new observation.

The detailed sensing procedure for Differential SED is as follows:

1) First, each $C R$ user locally calculates the difference between two consecutive energy observations. 
2) If this difference is less than the variable threshold, $\lambda_{d}$. The previously received energy observations will be taken into weight computation. In other words, CR users ignore the previous observation once the difference is greater than $\lambda_{d}$, as they think that PU has changed its state.

3) Similarly to the Two-Stage SED, in order to avoid CR users relying on too many past observations, we limit the number of past observations to be taken in to weight computation to a certain number. CR users then apply equal weighting to all energy observations that are taken into weight computation. The weighted observations are then aggregated as given in (8).

4) Then, the rest of the sensing procedures follows the previously proposed Two-Stage SED scheme.

\section{ANALYSIS}

A detailed analysis for the proposed Weighted SED and Two-Stage SED scheme, as well as the generic form for probability of false alarm and detection in both schemes, are presented in this section.

\section{A. Cooperative Spectrum Sensing}

In cooperative spectrum sensing, CR users forward its local decision to the fusion center to make a final decision. When the OR-rule is used, the final decision of $H_{1}$ is made when at least one CR user reports an $H_{1}$ local decision. The cooperative probability of false alarm and detection, respectively $Q_{f}$ and $Q_{d}$, can be expressed as

$$
\begin{aligned}
& Q_{f}(\lambda)=1-\left(1-P_{f}(\lambda)\right)^{U} \\
& Q_{d}(\lambda)=1-\left(1-P_{d}(\lambda)\right)^{U}
\end{aligned}
$$

where $U$ is the number of cooperative CR users.

As the global decision in cooperative spectrum sensing is made using the OR-rule from local decisions, the subsequent analysis can then be simplified by only considering the $P_{f}$ and $P_{d}$ in a $\mathrm{CR}$ node.

\section{B. Primary User activity model}

In order to evaluate $P_{f}$ and $P_{d}$ for the proposed schemes, the probabilities related to PU activity needs to be analysed. First, let $P\left(H_{j}\right)$ denote the probability that $\mathrm{PU}$ is in $H_{j}$ state and $P\left(H_{j} \rightarrow H_{k}\right)$ denote the transitional probability that PU was in $H_{j}$ state and is then in $H_{k}$ state in the next sensing slot. The probabilities related to the PU's state and transition
TABLE I

S : SET OF PU'S State

\begin{tabular}{|c|c|c|c|c|}
\cline { 2 - 5 } \multicolumn{1}{c|}{} & PU's State & & Previous Slot & Current Slot \\
\hline \multirow{2}{*}{$\mathbf{S}_{\text {idle }}$} & $s_{0}$ & & $H_{0}$ & $H_{0}$ \\
\cline { 2 - 5 } & $s_{2}$ & & $H_{1}$ & $H_{0}$ \\
\hline \hline \multirow{2}{*}{$\mathbf{S}_{\text {active }}$} & $s_{1}$ & & $H_{0}$ & $H_{1}$ \\
\cline { 2 - 5 } & $s_{3}$ & & $H_{1}$ & $H_{1}$ \\
\hline
\end{tabular}

are defined as follows [26].

$$
\begin{aligned}
P\left(H_{0}\right) & =\frac{\alpha}{\alpha+\beta} \\
P\left(H_{1}\right) & =\frac{\beta}{\alpha+\beta} \\
P\left(H_{0} \rightarrow H_{0}\right) & =(1-\beta) \cdot P\left(H_{0}\right)=\frac{\alpha(1-\beta)}{\alpha+\beta} \\
P\left(H_{0} \rightarrow H_{1}\right) & =\beta \cdot P\left(H_{0}\right)=\frac{\alpha \beta}{\alpha+\beta} \\
P\left(H_{1} \rightarrow H_{0}\right) & =\alpha \cdot P\left(H_{1}\right)=\frac{\alpha \beta}{\alpha+\beta} \\
P\left(H_{1} \rightarrow H_{1}\right) & =(1-\alpha) \cdot P\left(H_{1}\right)=\frac{(1-\alpha) \beta}{\alpha+\beta} .
\end{aligned}
$$

In addition, PU's state in each sensing slot is analyzed here because the proposed techniques do not only consider the current sensing slot, but also the previous slots. Let $\mathbf{S}$ be a set of PU's state in the current and previous time-slots, such that $\mathbf{S}=\left[s_{0}, s_{1}, s_{2}, \ldots, s_{2^{N}-1}\right] . \mathbf{S}_{\text {idle }}$ and $\mathbf{S}_{\text {active }}$ defines a subset of $\mathbf{S}$ where the PU is respectively idle and active in the current sensing-slot, regardless of its state in previous slots. Consider two observations for illustration purposes, the relationship between each element of $\mathbf{S}_{\text {idle }}, \mathbf{S}_{\text {active }}$ and each PU's state is shown in Table I. Denoting $P\left(s_{i} \mid H_{j}\right)$ as the conditional probability of state $s_{i}$ given that the PU is in $H_{j}$ state in the current sensing slot, it can be shown that

$$
P\left(s_{0} \mid H_{0}\right)=P\left(H_{0} \rightarrow H_{0} \mid H_{0}\right)=(1-\beta)
$$

and similarly

$$
\begin{aligned}
& P\left(s_{1} \mid H_{1}\right)=\alpha \\
& P\left(s_{2} \mid H_{0}\right)=\beta \\
& P\left(s_{3} \mid H_{1}\right)=(1-\alpha) .
\end{aligned}
$$

\section{Weighted SED}

We analyse the probability of false alarm and detection for the Weighted SED scheme, where a fixed number of local observations from the energy detector are taken into the weighting process as in (8). The probability of false alarm and the probability of detection can be formulated as a summation of the probability that $\mathrm{CR}$ user makes an $H_{1}$ decision in each PU's state $\left(s_{i}\right)$

$$
\begin{aligned}
& P_{f, W S}(\lambda)=\sum_{\forall s_{i} \in \mathbf{S}_{i d l e}} P\left(T>\lambda \mid s_{i}\right) \cdot P\left(s_{i} \mid H_{0}\right) \\
& P_{d, W S}(\lambda)=\sum_{\forall s_{i} \in \mathbf{S}_{\text {active }}} P\left(T>\lambda \mid s_{i}\right) \cdot P\left(s_{i} \mid H_{1}\right)
\end{aligned}
$$


where $P\left(T>\lambda \mid s_{i}\right)$ is the probability that $T$ is greater than $\lambda$ given that PU is in $s_{i}$ state.

This generic form can be used to formulate these probabilities with any number of local observations. Here, for illustration purposes, we consider taking only two observations in the weighting process. According to the transitional probability in (13), $P_{f, W S}$ and $P_{d, W S}$ from (14) can be expanded into

$$
\begin{aligned}
P_{f, W S}(\lambda)= & (1-\beta) \cdot P\left(T>\lambda \mid s_{0}\right) \\
& +\beta \cdot P\left(T>\lambda \mid s_{2}\right) \\
P_{d, W S}(\lambda)= & \alpha \cdot P\left(T>\lambda \mid s_{1}\right) \\
& +(1-\alpha) \cdot P\left(T>\lambda \mid s_{3}\right) .
\end{aligned}
$$

In order to obtain these probabilities, the weighted sum of observations under different PU's state needs to be evaluated. For instance, in the $s_{1}$ case where PU's state has changed from idle to active, obtaining $P\left(T>\lambda \mid s_{1}\right)$ needs an integration for the PDF of $T$

$$
\begin{aligned}
P\left(T>\lambda \mid s_{1}\right) & =\int_{\lambda}^{\infty} \int_{0}^{t} f\left(y_{1} \mid H_{0}\right) \cdot f\left(y_{2} \mid H_{1}\right) d y_{2} d t \\
& =\int_{\lambda}^{\infty} \int_{0}^{t} f\left(2 t-y_{2} \mid H_{0}\right) \cdot f\left(y_{2} \mid H_{1}\right) d y_{2} d t
\end{aligned}
$$

where $t=\frac{1}{2}\left(y_{1}+y_{2}\right)$ is the a weighted sum of received energy from previous and current sensing slot.

As it is mathematically difficult to obtain exact closedform for $P_{f, W S}$ and $P_{d, W S}$, we derive the approximated versions here. It can be noted that the term $P\left(T>\lambda \mid s_{i}\right)$ is highly dependent on PU's state as the received energy follows different PDF when PU is idle and active. Hence, we need to consider this probability case by case.

First, consider the $s_{0}$ case where PU is idle $\left(H_{0}\right)$ in both current and previous sensing slots.

$$
P\left(T>\lambda \mid s_{0}\right)=P\left(\frac{\left(y_{1}+y_{2}\right)}{2}>\lambda\right)=P\left(y_{1}+y_{2}>2 \lambda\right) \text {. }
$$

Both $y_{1}$ and $y_{2}$ here follow central chi-squared distribution with $2 m$ degree of freedom as shown in (2). From this, $y_{1}+$ $y_{2}$ also follows Chi-squared distribution with $4 m$ degree of freedom. Hence, $P\left(T>\lambda \mid s_{0}\right)$ can be approximated as

$$
P\left(T>\lambda \mid s_{0}\right) \approx \frac{\Gamma(2 m, \lambda)}{\Gamma(2 m)} .
$$

Then, for $s_{1}$ case, $\mathrm{PU}$ is idle in previous sensing slot and then becomes active in current slot. The received energy $y_{1}, y_{2}$ here follows $f\left(y \mid H_{0}\right)$ and $f\left(y \mid H_{1}\right)$ as in (2) and (6) respectively. However, it was shown in (16) that the closed form is difficult to obtain. Here, we adapt the central limit theorem and approximate that $y_{1}, y_{2}$ follows Gaussian distribution where $y_{1} \sim \mathcal{N}(2 m, 4 m)$ and $y_{2} \sim$ $\mathcal{N}\left(2 m(1+\bar{\gamma}), 4 m(1+\bar{\gamma})^{2}\right)$ [27]. From this, $y_{1}+y_{2}$ can be approximated as $\mathcal{N}\left(2 m(2+\bar{\gamma}), 4 m+4 m(1+\bar{\gamma})^{2}\right)$. Hence, $P\left(T>\lambda \mid s_{1}\right)$ can be approximated as a complementary cumulative density function (CCDF) for Gaussian distribution.

$$
P\left(T>\lambda \mid s_{1}\right) \approx \frac{1}{2} \operatorname{erfc}\left(\frac{\lambda-m(2+\bar{\gamma})}{\sqrt{2 \cdot\left(m+m(1+\bar{\gamma})^{2}\right)}}\right) .
$$

The approximation for $s_{2}$ case is similar to the above in $s_{1}$ case because PU is active in one slot and idle in the other slot. Hence, $P\left(T>\lambda \mid s_{2}\right)$ also follows (19).

Finally, PU is active in both current and previous sensing slots for $s_{3}$ case. Considering that Doppler frequency and channel variation is low, the channel gain for $y_{1}$ and $y_{2}$ would approximately be the same. It is approximated that $y_{1}+y_{2}$ follows (6) with extended time-bandwidth product of $2 \mathrm{~m}$. Hence, $P\left(T>\lambda \mid s_{3}\right)$ can be approximated as a modified version of probability of detection in (7), with time-bandwidth product of $2 m$ and detection threshold of $2 \lambda$.

\section{Two-Stage SED}

Here, we analyse the probability of false alarm and detection for Two-Stage SED scheme, $P_{f, T S}$ and $P_{d, T S}$. In this scheme, $\mathrm{CR}$ users adaptively determine the number of past observations based on the sensing result in the first stage. Hence, in addition to considering a set of PU's state $(\mathbf{S})$, the CR's first-stage decision also needs to be considered. Let $\hat{\mathbf{S}}$ be a set of CR's decision from the first stage in the current and previous timeslots, such that $\hat{\mathbf{S}}=\left[\hat{s_{0}}, \hat{s_{1}}, \hat{s_{2}}, \ldots, \hat{s}_{2^{N}}-1\right]$, which is defined similarly to $\mathbf{S}$ in Table I. First, let $P\left(H_{i} \mid H_{j}\right)$ be the probability that $\mathrm{CR}$ makes its first stage $H_{i}$ decision when PU is in $H_{j}$ state.

$$
\begin{aligned}
& F=P\left(H_{1} \mid H_{0}\right)=P_{f}\left(\lambda_{1}\right) \\
& D=P\left(H_{1} \mid H_{1}\right)=P_{d}\left(\lambda_{1}\right) .
\end{aligned}
$$

$P_{f}$ and $P_{d}$ for Two-Stage SED scheme can be formulated in a generic form as

$$
\begin{aligned}
& P_{f, T S}\left(\lambda_{2}\right)=\sum_{\forall s_{i} \in \mathbf{S}_{\text {idle }}, \forall \hat{s_{j}} \in \hat{\mathbf{S}}} P\left(T>\lambda_{2} \mid s_{i}, \hat{s_{j}}\right) \cdot P\left(s_{i}, \hat{s_{j}} \mid H_{0}\right) \\
& P_{d, T S}\left(\lambda_{2}\right)=\sum_{\forall s_{i} \in \mathbf{S}_{\text {active }}, \forall \hat{s_{j}} \in \hat{\mathbf{S}}} P\left(T>\lambda_{2} \mid s_{i}, \hat{s_{j}}\right) \cdot P\left(s_{i}, \hat{s_{j}} \mid H_{1}\right)
\end{aligned}
$$

where $\lambda_{2}$ is the detection threshold in the second stage. Similarly to the analysis for Weighted-SED scheme, this generic form can be used to formulate the probability of false alarm and detection with any number of local observations. For instance, assuming that a maximum of two observations are allowed in the second stage, $P_{f, T S}$ and $P_{d, T S}$ can be expanded as follows according to the probability of PU's transition in (13) and the probability related to the first-stage detection in (20).

$$
\begin{aligned}
P_{f, T S}\left(\lambda_{2}\right)= & (1-F) \cdot(1-F) \cdot(1-\beta) \cdot P\left(T>\lambda_{2} \mid s_{0}, \hat{s_{0}}\right) \\
& +(1-F) \cdot F \cdot(1-\beta) \cdot P\left(T>\lambda_{2} \mid s_{0}, \hat{s_{1}}\right) \\
& +F \cdot(1-F) \cdot(1-\beta) \cdot P\left(T>\lambda_{2} \mid s_{0}, \hat{s_{2}}\right) \\
& +F \cdot F \cdot(1-\beta) \cdot P\left(T>\lambda_{2} \mid s_{0}, \hat{s_{3}}\right) \\
& +(1-D) \cdot(1-F) \cdot \beta \cdot P\left(T>\lambda_{2} \mid s_{2}, \hat{s_{0}}\right) \\
& +(1-D) \cdot F \cdot \beta \cdot P\left(T>\lambda_{2} \mid s_{2}, \hat{s_{1}}\right) \\
& +D \cdot(1-F) \cdot \beta \cdot P\left(T>\lambda_{2} \mid s_{2}, \hat{s_{2}}\right) \\
& +D \cdot F \cdot \beta \cdot P\left(T>\lambda_{2} \mid s_{2}, \hat{s_{3}}\right) .
\end{aligned}
$$




$$
\begin{aligned}
P_{d, T S}\left(\lambda_{2}\right)= & (1-F) \cdot(1-D) \cdot \alpha \cdot P\left(T>\lambda_{2} \mid \hat{s_{1}}, \hat{s_{0}}\right) \\
& +(1-F) \cdot D \cdot \alpha \cdot P\left(T>\lambda_{2} \mid s_{1}, \hat{s_{1}}\right) \\
& +F \cdot(1-D) \cdot \alpha \cdot P\left(T>\lambda_{2} \mid s_{1}, \hat{s_{2}}\right) \\
& +F \cdot D \cdot \alpha \cdot P\left(T>\lambda_{2} \mid s_{1}, \hat{s_{3}}\right) \\
& +(1-D) \cdot(1-D) \cdot(1-\alpha) \cdot P\left(T>\lambda_{2} \mid s_{3}, \hat{s_{0}}\right) \\
& +(1-D) \cdot D \cdot(1-\alpha) \cdot P\left(T>\lambda_{2} \mid s_{3}, \hat{s_{1}}\right) \\
& +D \cdot(1-D) \cdot(1-\alpha) \cdot P\left(T>\lambda_{2} \mid s_{3}, \hat{s_{2}}\right) \\
& +D \cdot D \cdot(1-\alpha) \cdot P\left(T>\lambda_{2} \mid s_{3}, \hat{s_{3}}\right) .
\end{aligned}
$$

The $P_{f, T S}$ and $P_{d, T S}$ shown above is in a fully-expanded generic form and it can be categorized into three special cases depending on the value of first-stage and second-stage threshold $\left(\lambda_{1}\right.$ and $\left.\lambda_{2}\right)$.

1) $\lambda_{2}$ less than $\lambda_{1}$ : Consider the term $P\left(T>\lambda_{2} \mid s_{i}, \hat{s_{1}}\right)$ from (22). As CR's decision $\hat{s_{1}}$ is different between previous and current sensing slot, only then observation from the current slot is taken into second stage. With the first-stage decision being $H_{1}$ for current sensing slot, it can be deduced that the observation is larger than $\lambda_{1}$. Hence as $\lambda_{2}$ is less than $\lambda_{1}$, $P\left(T>\lambda_{2} \mid s_{i}, \hat{s_{1}}\right)$ is always 1 . Similarly $P\left(T>\lambda_{2} \mid s_{i}, \hat{s_{3}}\right)$ is also always 1 . Hence, $P_{f}$ and $P_{d}$ from (22) can be shortened into

$$
\begin{aligned}
P_{f, T S}\left(\lambda_{2}\right)= & F+(1-F) \cdot(1-F) \cdot(1-\beta) \cdot P\left(T>\lambda_{2} \mid s_{0}, \hat{s_{0}}\right) \\
& +F \cdot(1-F) \cdot(1-\beta) \cdot P\left(T>\lambda_{2} \mid s_{0}, \hat{s_{2}}\right) \\
& +(1-D) \cdot(1-F) \cdot \beta \cdot P\left(T>\lambda_{2} \mid s_{2}, \hat{s_{0}}\right) \\
& +D \cdot(1-F) \cdot \beta \cdot P\left(T>\lambda_{2} \mid s_{2}, \hat{s_{2}}\right)
\end{aligned}
$$

$$
\begin{aligned}
P_{d, T S}\left(\lambda_{2}\right)= & D+(1-F) \cdot(1-D) \cdot \alpha \cdot P\left(T>\lambda_{2} \mid s_{1}, \hat{s_{0}}\right) \\
& +F \cdot(1-D) \cdot \alpha \cdot P\left(T>\lambda_{2} \mid s_{1}, \hat{s_{2}}\right) \\
& +(1-D) \cdot(1-D) \cdot(1-\alpha) \cdot P\left(T>\lambda_{2} \mid s_{3}, \hat{s_{0}}\right) \\
& +D \cdot(1-D) \cdot(1-\alpha) \cdot P\left(T>\lambda_{2} \mid s_{3}, \hat{s_{2}}\right) .
\end{aligned}
$$

Then, consider state $\hat{s_{2}}$ where the decision from the previous and current sensing slot is different. Hence, only energy observation from the current slot is taken into the weighting process such that $T=Y$. With the first-stage decision being $H_{0}$ for current sensing slot, it can be deduced that the observation follows the truncated distribution from 0 to $\lambda_{1}$ and is always smaller than $\lambda_{1}$. Consequently, $P\left(T>\lambda_{2} \mid s_{i}, \hat{s_{2}}\right)$ in (23) analytically becomes

$$
\begin{aligned}
& P\left(T>\lambda_{2} \mid s_{i} \in \mathbf{S}_{\text {idle }}, \hat{s_{2}}\right)=\frac{P_{f}\left(\lambda_{2}\right)-P_{f}\left(\lambda_{1}\right)}{1-P_{f}\left(\lambda_{1}\right)} \\
& P\left(T>\lambda_{2} \mid s_{i} \in \mathbf{S}_{\text {active }}, \hat{s_{2}}\right)=\frac{P_{d}\left(\lambda_{2}\right)-P_{d}\left(\lambda_{1}\right)}{1-P_{d}\left(\lambda_{1}\right)} .
\end{aligned}
$$

Note that since $T$ follows a truncated distribution, the denominator of (24) is to normalize the probability by the overall possibility of $T$, i.e. probability that $T$ is less than $\lambda_{1}$.

Next, consider $P\left(T>\lambda_{2} \mid s_{i}, \hat{s_{0}}\right)$ where the decisions from the previous and current sensing slot are identical and two observations are taken into the weighting process. Here, $T$ follows a joint distribution of the two observations based on $s_{i}$. For example, in the $\left(s_{1}, \hat{s_{0}}\right)$ case, $T$ is a weighted summation from the two observations which respectively follow truncated $H_{0}$ in previous slot and truncated $H_{1}$ in current sensing slot. Hence, it is difficult to analytically obtain the term $P\left(T>\lambda_{2} \mid s_{1}, \hat{s_{0}}\right)$, as it involves various integrations of PDF for different truncated distributions as follows.

$$
f_{t}\left(t \mid s_{1}, \hat{s_{0}}\right)=\left\{\begin{array}{cc}
\int_{0}^{2 t} f\left(2 t-y_{2} \mid H_{0}\right) \cdot f\left(y_{2} \mid H_{1}\right) d y_{2} \\
, 0<t<\frac{\lambda_{1}}{2} \\
\int_{2 t-\lambda_{1}}^{\lambda_{1}} f\left(2 t-y_{2} \mid H_{0}\right) \cdot & f\left(y_{2} \mid H_{1}\right) d y_{2} \\
0 & , \frac{\lambda_{1}}{2}<t<\lambda_{1} \\
0 & , \text { otherwise. }
\end{array}\right.
$$

Then, to find the probability that $T$ is greater than $\lambda_{2}$, we need

$$
P\left(T>\lambda_{2}\right)=\int_{\lambda_{2}}^{\infty} f_{t}(t) d t .
$$

A closed form solution cannot be found and an approximated solution will be presented in Section IV-E.

2) $\lambda_{2}$ equals to $\lambda_{1}$ : When $\lambda_{2}$ equals to $\lambda_{1}, P(T>$ $\left.\lambda_{2} \mid s_{i}, \hat{s_{1}}\right)$ and $P\left(T>\lambda_{2} \mid s_{i}, \hat{s_{3}}\right)$ from (22) is always 1 as mentioned above. On the contrary, $P\left(T>\lambda_{2} \mid s_{i}, \hat{s_{0}}\right)$ and $P\left(T>\lambda_{2} \mid s_{i}, \hat{s_{2}}\right)$ are always 0 . Since the conditional state $\hat{s_{0}}$ and $\hat{s_{2}}$ requires $T$ to be smaller than $\lambda_{1}$, which equals to $\lambda_{2}$ in this case. Consequently, $P_{f, T S}$ and $P_{d, T S}$ can be shortened to

$$
\begin{aligned}
& P_{f, T S}\left(\lambda_{2}\right)=F=P_{f}\left(\lambda_{1}\right) \\
& P_{d, T S}\left(\lambda_{2}\right)=D=P_{d}\left(\lambda_{1}\right) .
\end{aligned}
$$

This result indicates that when $\lambda_{1}$ and $\lambda_{2}$ are the same, the Two-Stage SED will have identical performance with the conventional scheme.

3) $\lambda_{2}$ larger than $\lambda_{1}$ : Similar to the above, when $\lambda_{2}$ is larger than $\lambda_{1}, P\left(T>\lambda_{2} \mid s_{i}, \hat{s_{0}}\right)$ and $P\left(T>\lambda_{2} \mid s_{i}, \hat{s_{2}}\right)$ is always 0 as the observations are always smaller than $\lambda_{1}$ and $\lambda_{2}$.

Hence, $P_{f, T S}$ and $P_{d, T S}$ become

$$
\begin{aligned}
P_{f, T S}\left(\lambda_{2}\right)= & (1-F) \cdot F \cdot(1-\beta) \cdot P\left(T>\lambda_{2} \mid s_{0}, \hat{s_{1}}\right) \\
& +F \cdot F \cdot(1-\beta) \cdot P\left(T>\lambda_{2} \mid s_{0}, \hat{s_{3}}\right) \\
& +(1-D) \cdot F \cdot \beta \cdot P\left(T>\lambda_{2} \mid s_{2}, \hat{s_{1}}\right) \\
& +D \cdot F \cdot \beta \cdot P\left(T>\lambda_{2} \mid s_{2}, \hat{s_{3}}\right)
\end{aligned}
$$

$$
\begin{aligned}
P_{d, T S}\left(\lambda_{2}\right)= & (1-F) \cdot D \cdot \alpha \cdot P\left(T>\lambda_{2} \mid s_{1}, \hat{s_{1}}\right) \\
& +F \cdot D \cdot \alpha \cdot P\left(T>\lambda_{2} \mid s_{1}, \hat{s_{3}}\right) \\
& +(1-D) \cdot D \cdot(1-\alpha) \cdot P\left(T>\lambda_{2} \mid s_{3}, \hat{s_{1}}\right) \\
& +D \cdot D \cdot(1-\alpha) \cdot P\left(T>\lambda_{2} \mid s_{3}, \hat{s_{3}}\right) .
\end{aligned}
$$

For the decision state $\hat{s_{1}}$ here, where the decision from the previous and current sensing slot is also different, $P(T>$ 
$\left.\lambda_{2} \mid s_{i}, \hat{s_{1}}\right)$ terms in (28) then analytically becomes

$$
\begin{aligned}
& P\left(T>\lambda_{2} \mid s_{i} \in \mathbf{S}_{\text {idle }}, \hat{s_{1}}\right)=\frac{P_{f}\left(\lambda_{2}\right)}{P_{f}\left(\lambda_{1}\right)} \\
& P\left(T>\lambda_{2} \mid s_{i} \in \mathbf{S}_{\text {active }}, \hat{s_{1}}\right)=\frac{P_{d}\left(\lambda_{2}\right)}{P_{d}\left(\lambda_{1}\right)} .
\end{aligned}
$$

Finally, when the decision state is $\hat{s_{3}}$, two observations are weighted. As in the first case, $T$ follows a joint distribution from two truncated distributions and a closed form solution cannot be obtained.

\section{E. Two-Stage SED Approximation}

As it is mathematically intractable to obtain the exact closed-form for some terms in $P_{f, T S}$ and $P_{d, T S}$, we present an approximated approach for these terms where two obseravtions are in the weighting process. Similar to the Weighted SED scheme, we also adopt Gaussian distribution for the approximation. We further simplify the approximation by ignoring the truncation, and approximate $T$ as a summation of two Gaussian RVs. However, truncation is considered when evaluating the term $P\left(T>\lambda_{2} \mid s_{i}, \hat{s}_{i}\right)$. When $\lambda_{2}$ is less than $\lambda_{1}$,

$$
P\left(T>\lambda_{2} \mid s_{i}, \hat{s_{i}}\right) \approx \frac{F\left(\lambda_{2}\right)-F\left(\lambda_{1}\right)}{1-F\left(\lambda_{1}\right)}
$$

where $F($.$) is a CCDF function for a Gaussian distribution,$ and when $\lambda_{2}$ is larger than $\lambda_{1}$,

$$
P\left(T>\lambda_{2} \mid s_{i}, \hat{s_{i}}\right) \approx \frac{F\left(\lambda_{2}\right)}{F\left(\lambda_{1}\right)} .
$$

For instance, in $s_{1}$ and $\lambda_{2}$ is larger than $\lambda_{1}$ case, $T$ can be approximated as $\mathcal{N}\left(m(2+\bar{\gamma}), m+m(1+\bar{\gamma})^{2}\right)$. The CCDF becomes

$$
F(x)=\frac{1}{2} \operatorname{erfc}\left(\frac{x-m(2+\bar{\gamma})}{\sqrt{2 \cdot\left(m+m(1+\bar{\gamma})^{2}\right)}}\right) .
$$

\section{F. Differential SED}

Similar to the Two-Stage SED, Differential SED also adaptively determines the number of local observations. Hence, to analyse $P_{f, D i f f}$ and $P_{d, D i f f}$ for Differential SED, the probability that the difference between two consecutive observations is less than the threshold is needed. However, this involves the derivation of the PDF of the difference between two or more random variables, each of which is either $H_{0}$ or $H_{1}$ distributed. The analysis becomes mathematically un-trackable and for this reason, we do not analyze the Differential SED in this paper.

\section{Simulation Results}

The proposed schemes are evaluated using computer simulation with the following parameters. Three spatially separated users are considered for cooperative sensing. The timebandwidth product of 3 is set for each observation. Each packet has length of 100 symbols and the sampling frequency is 100 $k H z$. The PU's SNR is $3 \mathrm{~dB}$ and the channel is modelled as a time-varying Rayleigh fading channel with Doppler frequency



Fig. 5. Detection performance for Weighted SED scheme with three previous observations on optimal $H_{0}$ and optimal $H_{1}$ weighting vector, when PU is static

of $50 \mathrm{~Hz}$. For the two adaptive schemes, we set a maximum of three previous observations in the weighting process. Some parameters, which are specific to the particular proposed schemes, are mentioned within its own part.

\section{A. Weighted SED}

As shown in Section III-A, when the PU activity is static, equal weighting is the optimal weight for the $H_{0}$ case, but not for the $H_{1}$ case. Hence we first evaluate the performance for optimal weighting under these two cases. Then, the intermittent PU activity is modelled and the detection performance of the proposed Weighted SED with equal and exponential weighting is evaluated.

1) Optimal $H_{0}$ and $H_{1}$ weighting vector: To evaluate the weight vector for static PU activity, PU is modelled such that it is either always on or always off. The equal weighting $\mathbf{W}=\left[\begin{array}{llll}0.25 & 0.25 & 0.25 & 0.25\end{array}\right]$ is optimal when three previous observations are taken. However for the $H_{1}$ case, the set of optimal weighting vector which minimizes the variance of $H_{1}$ is $\mathbf{W}=\left[\begin{array}{llll}0.334 & 0.166 & 0.166 & 0.334\end{array}\right]$. The detection performance for the Weighted SED scheme in 50 $\mathrm{Hz}$ correlated channel with the optimized weight vectors is shown in Fig. 5, where $Q_{f}$ and $Q_{d}$ represent the probability of false alarm and detection for cooperative spectrum sensing. We model that it is equally probable for PU to be idle and active. The results show that the Weighted SED provides significantly better detection performance than the conventional cooperative OR-rule energy detection scheme. This is due to the use of past observations that are available locally. More importantly, the equal weighting is shown to outperform the weighting that minimizes the variance in $H_{1}$ case. This is highly advantageous as equal weighting is SNR and channel correlation independent, while the variance minimisation approach is not.

Then, intermittent PU activity is introduced and the detection performance from equal and exponential weighting vector are considered in Fig. 6. Similar to the above, we consider three past observations for Weighted SED scheme. 


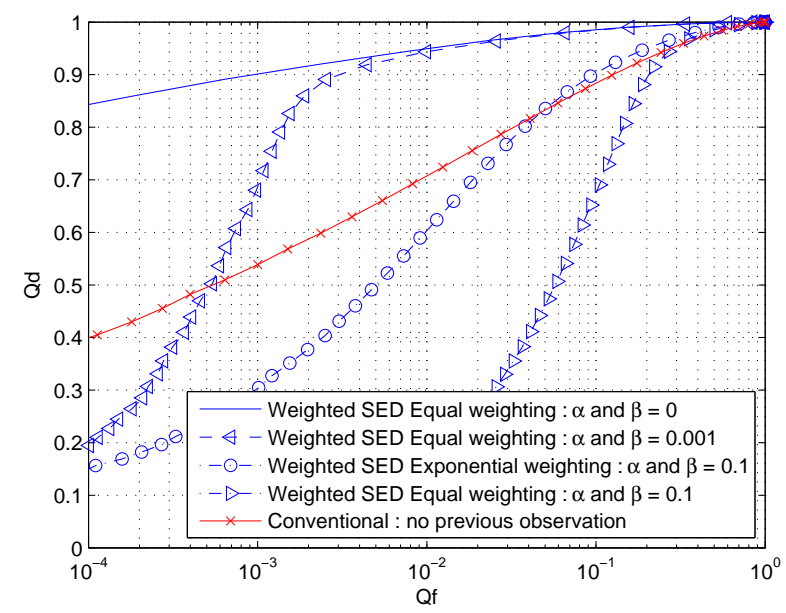

Fig. 6. Detection performance for Weighted SED scheme with static and intermittent PU activity in $50 \mathrm{~Hz}$ correlated channel

The PU activity $\alpha$ and $\beta$ are both set equal to 0.001 and 0.1 for the case that PU seldom and regularly changes its state respectively. First, we investigate the detection performance for the proposed Weighted SED scheme on equal weighting. Simulation result shows that the PU activity affects the detection performance. When PU activity is mostly static in the $\alpha$ and $\beta$ equal to 0.001 case, the proposed Weighted SED scheme with equal weighting is better than the conventional technique in high false alarm region, but still worse than the conventional technique in low false alarm region. However, when $\alpha$ and $\beta$ equal to 0.1 , the detection performance is degraded and it is even worse than the conventional energy detection technique which does not take any previous observation. This is because when the PU activity varies a lot, equal weighting relied too heavily on past observation that is already outdated. Hence, it is better to simply use the current observation. On the other hand, the result shows that the proposed exponential weighting provides an improvement on detection performance when $\alpha$ and $\beta$ equal to 0.1 , and even outperforms the conventional scheme in the high false alarm area. By applying exponential weighting, the more recent observations get higher weighting than the older observation, while the equal weighting one gives the same weight for every observation. Therefore, the performance is dependent on a proper weighting based on the PU activity. It is not desirable as the PU activity parameters $\alpha$ and $\beta$ are difficult to obtain in practice. On the contrary, the Two-Stage SED and Differential SED are both independent of these parameters.

\section{B. Two-Stage SED}

Here, we evaluate the detection performance for the proposed Two-Stage SED scheme, where a maximum of three previous observations are taken. Other simulation parameters are set up similarly to the Weighted SED scheme above. The PU activity is modelled with high variation such that $\alpha$ and $\beta$ is 0.1 . The detection performance for the proposed Two-Stage SED is evalutaed and compared to the Weighted SED scheme on equal weighting with one previous observation, which has



Fig. 7. Detection performance for the Two-Stage SED scheme in $50 \mathrm{~Hz}$ correlated channel case

best performance, and the conventional scheme. The detection performances for these schemes are shown in Fig. 7.

Simulation results show that the detection performance is improved in the high probability of detection area, in which the spectrum sensing procedure is expected to operate. In this scheme, there is a dip on the receiver operating characteristic (ROC) curve, at that point the detection performance is the same as conventional scheme. This occurs when the value for two thresholds $\left(\lambda_{1}\right.$ and $\left.\lambda_{2}\right)$ are the same. The reason for this behavior was analytically explained in Section IV-D. Besides, in terms of the sensing requirement under this simulation settings, the Two-Stage SED can meet the IEEE 802.22 spectrum sensing requirement on the probability of false alarm (10\%) and detection (90\%) [28], while the conventional and Weighted SED scheme can not.

\section{Differential SED}

The detection performance for the proposed Differential SED is shown in Fig. 8. This scheme can provide an improvement on detection performance in high probability of detection area, but without a performance dip as occurred in the TwoStage SED scheme. It also outperforms both the Weighted SED and the conventional scheme. Moreover, similar to the Two-Stage SED, the Differential SED can also meet the IEEE 802.22 spectrum sensing requirement under these settings, while Weighted SED and conventional scheme fail to do so.

\section{SNR requirement for IEEE 802.22 standard}

With both the Two-Stage SED and Differential SED performing similarly in the previous simulation setting, we compare them in terms of the minimum required SNR to achieve the IEEE 802.22 target $10 \% \quad Q_{f}$ and $90 \% Q_{d}$ in different scenarios of PU activity. The simulation parameters are set up similarly to the previous part. All three proposed schemes are compared to the conventional single threshold scheme, and their performances shown in Fig. 9. The result shows that the Weighted SED provides an advantage over the conventional 


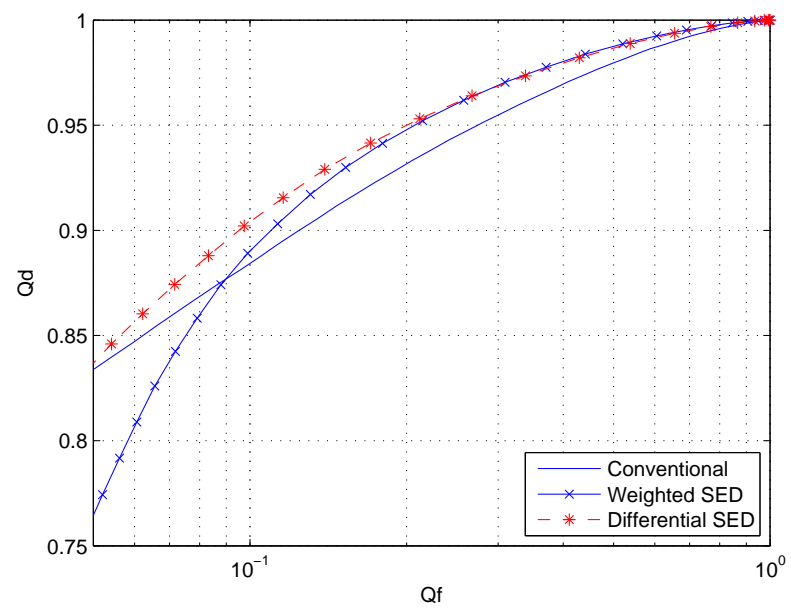

Fig. 8. Detection performance for the Differential SED scheme in $50 \mathrm{~Hz}$ correlated channel case

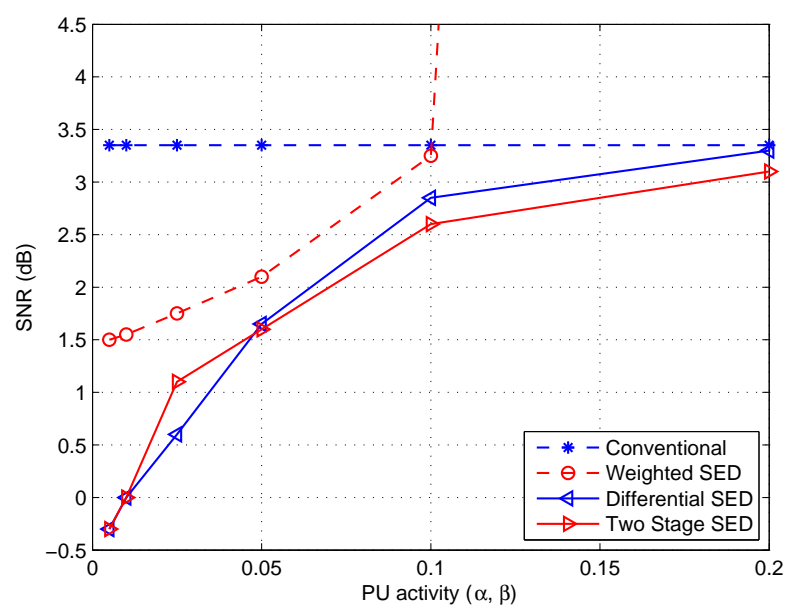

Fig. 9. Minimum required SNR for target probability of false alarm and detection

scheme when PU activity is low. However, in the case that PU activity is high, its performance significantly degrades as it still relies on the previous outdated observation.

On the other hand, the Two-Stage SED outperforms the Weighted SED and conventional scheme. In case when PU activity is low, high performance gain can be achieved. For instance, when $\alpha$ and $\beta$ is 0.01 , SNR needs to be at least $3.35 \mathrm{~dB}$ for the conventional scheme to achieve the detection performance target, while it is only $0 \mathrm{~dB}$ for the Two-Stage SED and Differential SED. Furthermore, in case when PU activity is high such as $\alpha$ and $\beta$ is 0.1 , only 2.6 and 2.85 $\mathrm{dB}$ of SNR is required respectively for the Two-Stage SED and Differential SED to achieve the detection target, while conventional scheme requires $3.35 \mathrm{~dB}$. It can also be seen that as the PU activity increases, the Two-Stage SED slightly outperforms the Differential SED.



Fig. 10. Probability of false alarm and detection in Weighted SED scheme

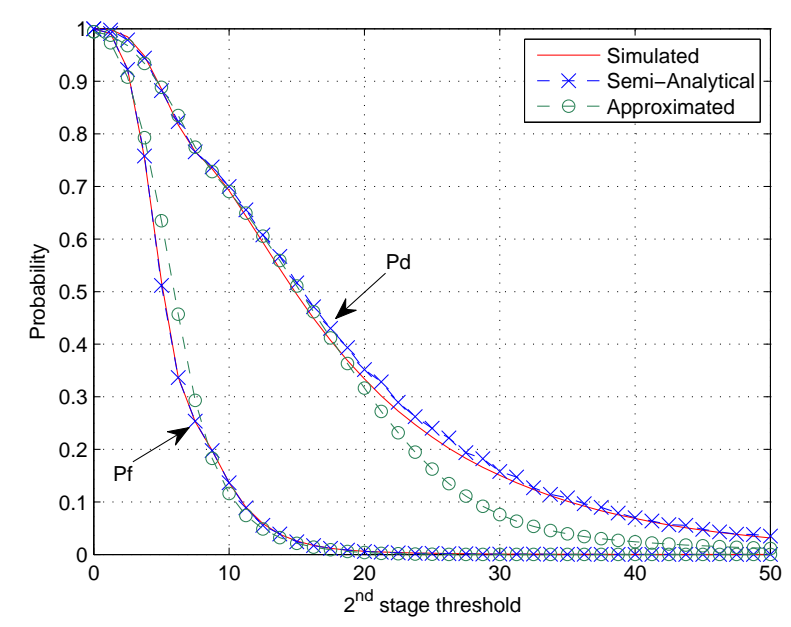

Fig. 11. Probability of false alarm and detection in Two-Stage SED scheme

\section{E. Analysis for probability of false alarm and detection}

In Section IV-C, the approximated probability of false alarm and detection for Weighted SED and Two-Stage SED schemes are derived. In this part, we compare them to the simulation results as well as the semi-analytical results obtained from numerically evaluating the terms that cannot be derived.

1) Weighted SED: Fig. 10 shows the semi-analytical result and the approximated $P_{f}$ and $P_{d}$ for Weighted SED scheme with two local observations equally weighted in the weighting process, and compare them with $P_{f}$ and $P_{d}$ obtained entirely from the simulation. PU acitivity is modelled with $\alpha$ and $\beta$ set to be 0.1 . The results show that the semi-analytical results on both probabilities closely match with those entirely from simulation. In addition, the approximation is also shown to be accurate.

2) Two-Stage SED: Unlike the Weighted SED scheme where only PU's state is considered, the analysis here considers both PU's state and CR's decision in the first-stage sensing. Fig. 11 show $P_{f}$ and $P_{d}$ in Two-Stage SED scheme with a maximum of two local observations in the weighting 
process and compare them with $P_{f}$ and $P_{d}$ obtained entirely from simulation. The results show that both probability of false alarm and detection obtained from the semi-analytical approach match closely with those from simulation. Moreover, the approximation is also shown to be accurate in high $P_{d}$ and low $P_{f}$ region, within which the CR system is expected work on.

\section{CONCLUSION}

In this paper, we propose three sequential cooperative spectrum sensing techniques. By taking energy observations in previous sensing slots, these schemes can exploit the time varying nature of the channel. The results showed that the proposed Weighted SED, Two-Stage SED and Differential SED can improve the detection performance. Especially in the Two-Stage SED and Differential SED, by adaptively relying on the past observations and the CR's estimation on the PU's state, further improvement on detection performance can be achieved. Moreover, both schemes are shown to provide a performance gain on minimum required SNR to achieve the IEEE 802.22 standard of $10 \%$ false alarm and $90 \%$ detection. When PU's activity is low, a significant gain can be achieved by using the adaptive schemes. Effectively, this allows the CR system to work in the scenario that conventional techniques can not accommodate the requirements. Furthermore, the probability of false alarm and detection in Weighted SED and Two-Stage SED schemes are derived. The approximated version for these schemes is also given and shown to match with the simulated result.

\section{APPENDIX}

The variance of $T$ is

$$
\begin{aligned}
\operatorname{Var}(T)= & \operatorname{Var}\left(\sum_{i=0}^{N-1} w_{i} Y_{i+N-1}\right) \\
= & \sum_{i=0}^{N-1} w_{i}^{2} \cdot \operatorname{Var}\left(Y_{i+N-1}\right) \\
& +2 \sum_{i, j ; i<j} w_{i} w_{j} \cdot \operatorname{Cov}\left(Y_{i+N-1}, Y_{j+N-1}\right) .
\end{aligned}
$$

In quasi-static fading channel, observation in each frame is independent to that in the previous and next frame. Hence $\operatorname{Cov}\left(Y_{i}, Y_{j}\right)=0$ for all $i$ and $j$. Then,

$$
\operatorname{Var}(T)=\sum_{i=0}^{N-1} w_{i}^{2} \cdot \operatorname{Var}\left(Y_{i+N-1}\right) .
$$

For equal weighting, the weighting vector $\mathbf{W}=$ $\left[\begin{array}{llll}\frac{1}{N} & \frac{1}{N} & \ldots & \frac{1}{N}\end{array}\right]$ and for each $Y_{i+N-1}$, its variance is the same such that $\operatorname{Var}(Y)=\operatorname{Var}\left(Y_{N-1}\right)=\ldots=\operatorname{Var}\left(Y_{0}\right)$. Hence,

$$
\operatorname{Var}(T)=\frac{\operatorname{Var}(Y)}{N} .
$$

This concludes that when equal weighting is applied under quasi-static channel, the variance of $T$ decreases linearly with increasing $N$.

\section{REFERENCES}

[1] I. Akyildiz, W. Lee, M. Vuran, and S. Mohanty, "Next generation/dynamic spectrum access/cognitive radio wireless networks: a survey," Computer Networks, vol. 50, no. 13, pp. 2127-2159, 2006.

[2] "FCC frees up vacant TV airwaves for super wi-fi, technologies and other technologies,," 2011. [Online]. Available: http://www.fcc.gov

[3] White Paper, Nokia Siemens Networks, "2020: Beyond 4G radio evolution for the gigabit experience," 2011.

[4] J. Mitola III, "Software radios: Survey, critical evaluation and future directions," IEEE Aerospace and Electronic Systems Magazine, vol. 8 , no. 4, pp. 25-36, 1993.

[5] S. Dixit, "Future of IMT systems: Wireless world vision 2020 - ITU," 2013. [Online]. Available: http://www.itu.int/

[6] T. Yucek and H. Arslan, "A survey of spectrum sensing algorithms for cognitive radio applications," IEEE Communications Surveys \& Tutorials, vol. 11, no. 1, pp. 116-130, 2009.

[7] K. Ben Letaief and W. Zhang, "Cooperative communications for cognitive radio networks," Proceedings of the IEEE, vol. 97, no. 5, pp. 878-893, 2009.

[8] S. Haykin, D. J. Thomson, and J. H. Reed, "Spectrum sensing for cognitive radio," Proceedings of the IEEE, vol. 97, no. 5, pp. 849-877, 2009.

[9] E. Axell, G. Leus, E. G. Larsson, and H. V. Poor, "Spectrum sensing for cognitive radio: State-of-the-art and recent advances," Signal Processing Magazine, IEEE, vol. 29, no. 3, pp. 101-116, 2012.

[10] G. Ganesan and Y. Li, "Cooperative spectrum sensing in cognitive radio networks," in Proc. IEEE Dynamic Spectrum Access Networks, pp. 137143,2005

[11] J. Zhu, Z. Xu, F. Wang, B. Huang, and B. Zhang, "Double threshold energy detection of cooperative spectrum sensing in cognitive radio," in Cognitive Radio Oriented Wireless Networks and Communications, 2008. CrownCom 2008. 3rd International Conference on, may 2008, pp. $1-5$.

[12] F. Liu, J. Wang, and Y. Han, "An adaptive double thresholds scheme for spectrum sensing in cognitive radio networks," in Signal Processing, Communication and Computing (ICSPCC), 2013 IEEE International Conference on, 2013, pp. 1-5.

[13] F. Mohammed and M. Deriche, "A two-threshold cooperative spectrum sensing algorithm using swarm intelligence," in Computing, Communications and IT Applications Conference (ComComAp), 2013, 2013, pp. $59-62$.

[14] G. Li, A. Cano, and S. Zhu, "High-diversity cooperative spectrum sensing in cognitive radio networks," in Global Telecommunications Conference (GLOBECOM 2010), 2010 IEEE. IEEE, 2010, pp. 1-5.

[15] H. Hu, L. Wu, N. Li, D. Zhang, and Y. Xu, "Efficient cooperative spectrum sensing with minimum sensing error in cognitive radio networks," in Wireless Communications Signal Processing (WCSP), 2012 International Conference on, 2012, pp. 1-6.

[16] A. Singh, M. R. Bhatnagar, and R. K. Mallik, "Cooperative spectrum sensing in multiple antenna based cognitive radio network using an improved energy detector," Communications Letters, IEEE, vol. 16, no. 1 , pp. 64-67, 2012.

[17] _ - "Performance analysis of multiple sample based improved energy detector in collaborative CR networks," in Personal Indoor and Mobile Radio Communications (PIMRC), 2013 IEEE 24th International Symposium on. IEEE, 2013, pp. 2728-2732.

[18] S. Mitsuya, K. Kobayashi, T. Yamazato, and M. Katayama, "Improvement of sequential-test-based cooperative spectrum sensing systems in band limited control channels," in Proc. IEEE Intl. Symp. Communications and Information Technologies, pp. 968-973, 2010.

[19] W. Zhang, R. Mallik, and K. Letaief, "Optimization of cooperative spectrum sensing with energy detection in cognitive radio networks," IEEE Transactions on Wireless Communications, vol. 8, no. 12, pp. 5761-5766, 2009.

[20] H. Urkowitz, "Energy detection of unknown deterministic signals," in Proceedings of the IEEE, vol. 55, no. 4, pp. 523-531, 1967.

[21] A. Ghasemi and E. Sousa, "Opportunistic spectrum access in fading channels through collaborative sensing," Journal of Communications, vol. 2, no. 2, p. 71, 2007

[22] I. Gradshteyn, I. Ryzhik, A. Jeffrey, and D. Zwillinger, Table of integrals, series, and products. Academic press, 2007.

[23] M. Khoshkholgh, K. Navaie, and H. Yanikomeroglu, "On the impact of the primary network activity on the achievable capacity of spectrum sharing over fading channels," IEEE Transactions on Wireless Communications, vol. 8, no. 4, pp. 2100-2111, 2009. 
[24] J. Proakis and D. Manolakis, "Digital signal processing, principles. algorithms, and applications, printice-hall," Inc, New Jersey, 1996.

[25] F. Digham, M. Alouini, and M. Simon, "On the energy detection of unknown signals over fading channels," in Communications, 2003. ICC'03. IEEE International Conference on, vol. 5. Ieee, 2003, pp. $3575-3579$.

[26] O. Ibe, Markov processes for stochastic modeling. Academic press, 2008.

[27] D. Cabric, A. Tkachenko, and R. W. Brodersen, "Experimental study of spectrum sensing based on energy detection and network cooperation," in Proceedings of the first international workshop on Technology and policy for accessing spectrum. ACM, 2006, p. 12.

[28] C. Stevenson, G. Chouinard, Z. Lei, W. Hu, S. Shellhammer, and W. Caldwell, "IEEE 802.22: The first cognitive radio wireless regional area network standard," IEEE Communications Magazine, vol. 47, no. 1, pp. 130-138, 2009.



Warit Prawatmuang received the BEng degree in Computer Engineering (First Class Honor) from Chiang Mai University, Thailand in 2008, the MSc degree in Communication Engineering (Distinction) in 2009 and the $\mathrm{PhD}$ degree in Electrical and Electronic Engineering in 2013, both from the University of Manchester, UK.

His research interests include cognitive radio, spectrum sensing and green communications. Dr. Prawatmuang has served as a reviewer for several IEEE conferences and letters.

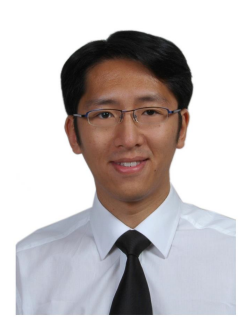

Daniel K. C. So (S'96-M'97-S'98-M'03) received the BEng degree in Electrical and Electronics Engineering (First Class Honor) from the University of Auckland, New Zealand in 1996, and the PhD degree in Electrical and Electronics Engineering from the Hong Kong University of Science and Technology (HKUST), Hong Kong in 2003.

From 1997 to 1998, he joined the Orion Systems International Limited, New Zealand, as a software engineer, and was later promoted to senior software engineer. Since 2003, he has been a lecturer and now a senior lecturer in the School of Electrical and Electronics Engineering, The University of Manchester, UK.

His research interests includes green communications, heterogeneous networks, cognitive radio, MIMO systems, cooperative MIMO schemes, multihop communication, OFDM, single carrier FDMA, multicarrier CDMA, channel equalization and estimation techniques.He regularly serves on the technical program committee of major international conferences.



Emad Alsusa (M'06-SM'07) received the Ph.D. degree in electrical and electronic engineering from Bath University, UK, in 2000 . He then joined Edinburgh University, as a MobileVCE Postdoctoral Research Fellow, working on link enhancement techniques for future high data rate wireless communication systems. In 2003, he joined the University of Manchester as an academic member of the School of Electrical and Electronic Engineering, where he lectures on communication engineering subjects.

His research interests include signal processing techniques and analysis of wireless communication networks, with particular focus on cognitive radio, interference mitigation, multiuser MIMO, GreenComm and energy and spectrum optimization techniques. Dr. Alsusa served as a Technical Program Committee member on numerous IEEE flagship conferences and chaired the Manchester EEE postgraduate conference in 2010. 